\title{
Accelerating adiabatic quantum transfer for three-level $\Lambda$-type structure systems via picture transformation
}

\author{
Yi-Hao Kang ${ }^{1,2}$, Qi-Cheng $\mathrm{Wu}^{1,2}$, Ye-Hong $\mathrm{Chen}^{1,2}$, \\ Zhi-Cheng Shi ${ }^{1,2}$, Jie Song ${ }^{3}$, and Yan Xia ${ }^{1,2, *}$ \\ ${ }^{1}$ Department of Physics, Fuzhou University, Fuzhou 350116, China \\ ${ }^{2}$ Fujian Key Laboratory of Quantum Information and \\ Quantum Optics (Fuzhou University), Fuzhou 350116, China \\ ${ }^{3}$ Department of Physics, Harbin Institute of Technology, Harbin 150001, China \\ In this paper, we investigate the quantum transfer for the system with three- \\ level $\Lambda$-type structure, and construct a shortcut to the adiabatic passage via picture \\ transformation to speed up the evolution. We can design the pulses directly without \\ any additional couplings. Moreover, by choosing suitable control parameters, the \\ Rabi frequencies of pulses can be expressed by the linear superpositions of Gaussian \\ functions, which could be easily realized in experiments. Compared with the previous \\ works using the stimulated Raman adiabatic passage, the quantum transfer can be \\ significantly accelerated with the present scheme.
}

PACS numbers: 03.67. Hk, 03.65. Ud

Keywords: Shortcut to adiabatic passage; Picture transformation; Three-level $\Lambda$-type system

\section{INTRODUCTION}

The three-level $\Lambda$-type system is known as a very important model in quantum information processing (QIP). Many quantum information tasks, such as the preparations of entanglement and the operations of various quantum gates, can be implemented in physical systems which are equivalent or approximately equivalent to three-level systems with $\Lambda$-type structures [1 16]. It is universally known that, to manipulate states of a three-level quantum system with electromagnetic field, there exists two typical methods, the $\pi$-pulse [17, 18] and the adiabatic passage [19 23]. These two methods hold their own advantages,

\footnotetext{
* E-mail: xia-208@163.com
} 
but both reveal their shortcomings. The $\pi$-pulse allows physical systems evolve quickly, but the pulses should be controlled very accurately, which bring challenges to experiments in some cases. On the other hand, the adiabatic passage is famous for its robustness against the imperfect operations and the deviations of the control parameters, but badly limits the evolution speed of the systems, which makes the systems more sensitive to some kinds of noise and decoherence factors. For the sake of both high evolution speed and robustness, a new technique named "Shortcuts to adiabatic passage" (STAP) 24 33] has been proposed.

The STAP suggests the system evolving in a controllable nonadiabatic way, so that the adiabatic condition, which limits the evolution speed of the system, can be abandoned. Besides, when the boundary condition of the control parameters is well designed, the STAP is also robust against the imperfect operations and the deviations of the control parameters. Since the STAP combines the advantages of both the $\pi$-pulse and the adiabatic passage, it has attracted many interests of researches in different fields [34 56]. For example, Torrontegui et al. [41] have used STAP to transport Bose-Einstein condensates. Ruschhaupt et al. [43] have achieved a population inversion in a two-level quantum system with STAP. Among these schemes [24 56], the method named "transitionless quantum driving" (TQD) (also known as the "counterdiabatic driving") [24 26, 55, 56] is one of the famous methods for constructing STAP, whose idea is to cancel the nonadiabatic transitions between the eigenstates for the original Hamiltonian of the system by adding "counterdiabatic" (CD) terms. The CD terms can be calculated easily, and their mathematic expressions are usually not too complex. For example, Demirplak et al. [24] have first used counterdiabatic fields to accelerate adiabatic passages, and shown that a population transfer between molecular states could be perfectly achieved, which is a pioneering work of STAP. Moreover, Du et al. [55] have experimentally shown that TQD could be used to design pulses to construct STAP for cold atoms. Furthermore, TQD has also be exploited by An et al. [56] to experimentally realize trapped-ion displacement in phase space. However, the CD terms sometimes play the roles as the additional couplings which are hard to be realized in real experiments. For example, it is indicated in many previous schemes [31, 57 -59] that, for a three-level $\Lambda$-type atom, the CD terms are the special one-photon 1-3 pulse (the microwave field), which bring troubles to the experimental realization.

To overcome the difficulties of TQD, many interesting schemes [60-73] have been put forward. For example, Ibáñez et al. [66, 67] have pointed out that a sequence of shortcuts 
to adiabaticity can be built with similar way of TQD via iterative interaction pictures. Subsequently, this method was used in a three-level system with $\Lambda$-type structure by Song et al. [68]. They have shown that the difficulties of TQD can be overcome, and the STAP can be constructed by adjusting the Rabi frequencies of pulses in original Hamiltonian, so the additional couplings are unnecessary. Chen et al. [71] have also come up with an interesting idea to construct an experimentally feasible Hamiltonian for a three-level system by using multi-mode driving of a set of moving states. Baksic et al. [73] have proposed an interesting scheme to speed up the quantum transfer for a three-level system with a serial of dressed states. They have shown that canceling the transitions between the chosen dressed states instead of the transitions between the eigenstates of the original Hamiltonian can avoid the difficulties of TQD, and the extra couplings are also unnecessary.

Inspired by the works [60 73], we propose an alternative scheme to accelerate the quantum transfer for the system with three-level $\Lambda$-type structure. Different from previous schemes, we directly investigate the dynamics of the three-level $\Lambda$-type system and the solution of the Schrödinger equation with only one picture transformation. The relationships of several parameters are studied. By designing these parameters suitably, the Rabi frequencies of pulses can be directly given, and they can be expressed by the linear superpositions of Gaussian functions, which are feasible in experiments. Meanwhile, the additional couplings are not required. In the end of the paper, we perform the numerical simulations, which show that the present scheme is effective. What is more, the quantum transfer can be significantly accelerated by applying the scheme instead of that with the stimulated Raman adiabatic passage.

\section{ACCELERATING ADIABATIC QUANTUM TRANSFER FOR THREE-LEVEL $\Lambda$-TYPE STRUCTURE SYSTEM VIA PICTURE TRANSFORMATION}

In this section, we start to introduce the method of the present scheme. For a system with the three-level $\Lambda$-type structure, the Hamiltonian has the general form as

$$
H_{0}(t)=\Omega_{1}(t)(|1\rangle\langle 2|+| 2\rangle\langle 1|)+\Omega_{2}(t)(|3\rangle\langle 2|+| 2\rangle\langle 3|),
$$


where pulse with Rabi frequency $\Omega_{1}(t)\left(\Omega_{2}(t)\right)$ drives the transition $|1\rangle \leftrightarrow|2\rangle(|2\rangle \leftrightarrow|3\rangle)$. We suppose

$$
G_{1}=\left[\begin{array}{lll}
0 & 1 & 0 \\
1 & 0 & 0 \\
0 & 0 & 0
\end{array}\right], \quad G_{2}=\left[\begin{array}{lll}
0 & 0 & 0 \\
0 & 0 & 1 \\
0 & 1 & 0
\end{array}\right], \quad G_{3}=\left[\begin{array}{ccc}
0 & 0 & -i \\
0 & 0 & 0 \\
i & 0 & 0
\end{array}\right]
$$

which satisfy the commutation relations $\left[G_{1}, G_{2}\right]=i G_{3},\left[G_{2}, G_{3}\right]=i G_{1}$ and $\left[G_{3}, G_{1}\right]=i G_{2}$. Assuming $\Omega(t)=\sqrt{\Omega_{1}^{2}(t)+\Omega_{2}^{2}(t)}, \tan \theta(t)=\Omega_{1}(t) / \Omega_{2}(t)$, the Hamiltonian in Eq. (11) can be rewritten as

$$
H_{0}(t)=\Omega(t)\left(\sin \theta G_{1}+\cos \theta G_{2}\right) .
$$

As the system possesses $S U(2)$ symmetry, we perform a picture transformation as $\left|\psi_{1}(t)\right\rangle=B^{\dagger}(t)\left|\psi_{0}(t)\right\rangle$ with the unitary operator $B(t)=e^{-i \epsilon(t) G_{3}}$, where $\left|\psi_{0}(t)\right\rangle$ is the wave function in the original picture, $\left|\psi_{1}(t)\right\rangle$ is the wave function after the picture transformation, and $\epsilon(t)$ is a real parameter. With the picture transformation, $H_{0}(t)$ will be transformed into

$$
\begin{aligned}
H_{1}(t) & =B^{\dagger}(t) H_{0}(t) B(t)-i B^{\dagger}(t) \dot{B}(t) \\
& =\Omega\left(\sin \theta e^{i \epsilon G_{3}} G_{1} e^{-i \epsilon G_{3}}+\cos \theta e^{i \epsilon G_{3}} G_{2} e^{-i \epsilon G_{3}}\right)-\dot{\epsilon} G_{3} \\
& =\Omega \sin (\theta+\epsilon) G_{1}+\Omega \cos (\theta+\epsilon) G_{2}-\dot{\epsilon} G_{3} .
\end{aligned}
$$

In the following, we will prove that the Hamiltonian $H_{1}(t)$ in Eq. (4) can be generated by the evolution operator in form of $U_{1}(t)=e^{i \mu\left(\sin \varphi G_{1}+\cos \varphi G_{2}\right)}$ with parameters $\mu(t)$ and $\varphi(t)$.

At the beginning, we assume $M(t)=\left(\sin \varphi G_{1}+\cos \varphi G_{2}\right)$. The operator $M$ has three eigenstates

$$
\begin{aligned}
& \left|\xi_{0}\right\rangle=\cos \varphi|1\rangle-\sin \varphi|3\rangle, \\
& \left|\xi_{+}\right\rangle=\frac{1}{\sqrt{2}}(\sin \varphi|1\rangle+|2\rangle+\cos \varphi|3\rangle), \\
& \left|\xi_{-}\right\rangle=\frac{1}{\sqrt{2}}(\sin \varphi|1\rangle-|2\rangle+\cos \varphi|3\rangle),
\end{aligned}
$$


corresponding to the eigenvalues 0,1 and -1 , respectively. It is obviously that

$$
\begin{aligned}
& M(t)=\left|\xi_{+}\right\rangle\left\langle\xi_{+}|-| \xi_{-}\right\rangle\left\langle\xi_{-}\right|, \\
& M^{n}(t)=\left|\xi_{+}\right\rangle\left\langle\xi_{+}\left|+(-1)^{n}\right| \xi_{-}\right\rangle\left\langle\xi_{-}\right|, \\
& U_{1}(t)=e^{i \mu M}=\sum_{n=0}^{\infty} \frac{i^{n} \mu^{n} M^{n}}{n !}=\left|\xi_{0}\right\rangle\left\langle\xi_{0}\left|+e^{i \mu}\right| \xi_{+}\right\rangle\left\langle\xi_{+}\left|+e^{-i \mu}\right| \xi_{-}\right\rangle\left\langle\xi_{-}\right|,
\end{aligned}
$$

and

$$
\begin{aligned}
& \left|\dot{\xi}_{0}\right\rangle=-\frac{\dot{\varphi}}{\sqrt{2}}\left(\left|\xi_{+}\right\rangle+\left|\xi_{-}\right\rangle\right), \\
& \left|\dot{\xi}_{+}\right\rangle=\left|\dot{\xi}_{-}\right\rangle=\frac{\dot{\varphi}}{\sqrt{2}}\left|\xi_{0}\right\rangle .
\end{aligned}
$$

Therefore, we can further obtain

$$
\begin{aligned}
i \dot{U}_{1}(t) U_{1}^{\dagger}(t) & =-(\dot{\mu} \sin \varphi+\dot{\varphi} \sin \mu \cos \varphi) G_{1}+(\dot{\varphi} \sin \mu \sin \varphi-\dot{\mu} \cos \varphi) G_{2}-\dot{\varphi}(1-\cos \mu) G_{3} \\
& =\gamma \sin (\varphi-\delta-\pi / 2) G_{1}+\gamma \cos (\varphi-\delta-\pi / 2) G_{2}-\dot{\varphi}(1-\cos \mu) G_{3},
\end{aligned}
$$

where, $\gamma=\sqrt{\dot{\mu}^{2}+\dot{\varphi}^{2} \sin ^{2} \mu}$ and $\tan \delta=\dot{\mu} /(\dot{\varphi} \sin \mu)$. Comparing Eq. (8) with Eq. (44), we have

$$
\begin{aligned}
& \Omega=\gamma=\sqrt{\dot{\mu}^{2}+\dot{\varphi}^{2} \sin ^{2} \mu}, \\
& \theta+\epsilon=\varphi-\delta-\pi / 2, \\
& \dot{\epsilon}=\dot{\varphi}(1-\cos \mu) .
\end{aligned}
$$

On the other hand, we assume the initial time is $t_{i}=0$ and the final time is $t_{f}=T$. If $\left|\psi_{0}(0)\right\rangle=|1\rangle, \epsilon(0)=\varphi(0)=0$, we have $\left|\psi_{1}(0)\right\rangle=\left|\psi_{0}(0)\right\rangle=|1\rangle$. With the evolution operator $U_{1}(t)$, we obtain the wave function in the transformed picture as

$$
\begin{aligned}
\left|\psi_{1}(t)\right\rangle & =U_{1}(t)\left|\psi_{1}(0)\right\rangle \\
& =\left(\left|\xi_{0}\right\rangle\left\langle\xi_{0}\left|+e^{i \mu}\right| \xi_{+}\right\rangle\left\langle\xi_{+}\left|+e^{-i \mu}\right| \xi_{-}\right\rangle\left\langle\xi_{-}\right|\right)\left[\cos \varphi\left|\xi_{0}\right\rangle+\frac{1}{\sqrt{2}} \sin \varphi\left(\left|\xi_{+}\right\rangle+\left|\xi_{-}\right\rangle\right)\right]
\end{aligned}
$$




$$
\begin{aligned}
& =\cos \varphi\left|\xi_{0}\right\rangle+\frac{1}{\sqrt{2}} \sin \varphi\left(e^{i \mu}\left|\xi_{+}\right\rangle+e^{-i \mu}\left|\xi_{-}\right\rangle\right) \\
& =\left(\cos ^{2} \varphi+\sin ^{2} \varphi \cos \mu\right)|1\rangle+i \sin \varphi \sin \mu|2\rangle+\sin \varphi \cos \varphi(\cos \mu-1)|3\rangle
\end{aligned}
$$

Moving back to the original picture, the wave function is

$$
\begin{aligned}
\left|\psi_{0}(t)\right\rangle & =B(t)\left|\psi_{1}(t)\right\rangle \\
& =\left[\cos \epsilon\left(\cos ^{2} \varphi+\sin ^{2} \varphi \cos \mu\right)-\sin \epsilon \sin \varphi \cos \varphi(\cos \mu-1)\right]|1\rangle+i \sin \varphi \sin \mu|2\rangle+ \\
& {\left[\cos \epsilon \sin \varphi \cos \varphi(\cos \mu-1)+\sin \epsilon\left(\cos ^{2} \varphi+\sin ^{2} \varphi \cos \mu\right)\right]|3\rangle }
\end{aligned}
$$

With Eq. (9) and Eq. (11), we can design the control parameters $\varphi, \epsilon$ and $\mu$ to realize a quantum transfer with experimental feasible pulses.

As an example, we design a set of parameters and perform numerical simulations to show the effectiveness of the present scheme. For simplicity, we assume $\dot{\mu} \equiv 0$, so that $\kappa=1-\cos \mu$ is a constant $(0 \leq \kappa \leq 2)$. Then we have $\epsilon=\kappa \varphi, \Omega=|\dot{\varphi} \sin \mu|, \delta=0, \theta=(1-\kappa) \varphi-\pi / 2$ and the wave function in Eq. (11) will become

$$
\begin{aligned}
\left|\psi_{0}(t)\right\rangle= & {\left[\cos (\kappa \varphi)\left(1-\kappa \sin ^{2} \varphi\right)+\kappa \sin (\kappa \varphi) \sin \varphi \cos \varphi\right]|1\rangle+i \sqrt{2 \kappa-\kappa^{2}} \sin \varphi|2\rangle+} \\
& {\left[\sin (\kappa \varphi)\left(1-\kappa \sin ^{2} \varphi\right)-\kappa \cos (\kappa \varphi) \sin \varphi \cos \varphi\right]|3\rangle }
\end{aligned}
$$

Assuming that we desire a quantum transfer $|1\rangle \rightarrow|3\rangle$, we should have $2 \kappa-\kappa^{2}=0$ or $\varphi(T)=m \pi(m=0, \pm 1, \pm 2, \cdots)$. It is obviously that when $2 \kappa-\kappa^{2}=0$, which gives $\kappa=0$ or $\kappa=2$, the result is $\left|\psi_{0}(t)\right\rangle \equiv|1\rangle$. That means the quantum transfer can not be realized when $2 \kappa-\kappa^{2}=0$. Therefore, we select $\varphi(T)=\pi$ here. So we have

$$
\left|\psi_{0}(T)\right\rangle=\cos (\kappa \pi)|1\rangle+\sin (\kappa \pi)|3\rangle
$$

In order to realize the quantum transfer $|1\rangle \rightarrow|3\rangle$, we choose $\kappa=1 / 2$, i.e., $\mu=\pi / 3$. Then the following results can be obtained: $\Omega(t)=\frac{\sqrt{3}}{2}|\dot{\varphi}|, \theta(t)=\frac{\varphi-\pi}{2}$. For the sake of robustness against deviation of operation time, the boundary condition $\Omega(0)=\Omega(T)=0$ is advisable. Therefore, we choose

$$
\varphi(t)=\frac{\pi}{2}\left[1-\cos \left(\frac{\pi t}{T}\right)\right], \quad \dot{\varphi}(t)=\frac{\pi^{2}}{2 T} \sin \left(\frac{\pi t}{T}\right)
$$



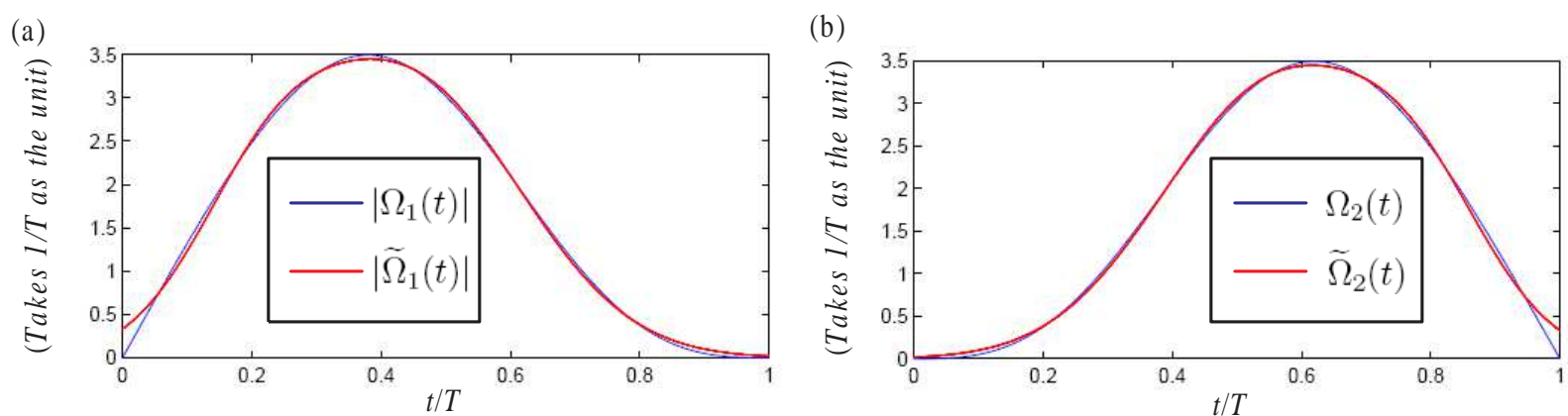

FIG. 1: (a) Comparison between $\left|\Omega_{1}(t)\right|$ and $\left|\widetilde{\Omega}_{1}(t)\right|$ (versus $t / T$ ). (b) Comparison between $\Omega_{2}(t)$ and $\widetilde{\Omega}_{2}(t)($ versus $t / T)$.

Until now, the only question remained is that the Rabi frequencies $\Omega_{1}(t)=\Omega(t) \sin \theta(t)$ and $\Omega_{2}(t)=\Omega(t) \cos \theta(t)$ are still too complex for the experimental realization. For the sake of the experimental feasibility, we apply the curve fitting to $\Omega_{1}(t)$ and $\Omega_{2}(t)$, and obtain two replacing Rabi frequencies $\widetilde{\Omega}_{1}(t)$ and $\widetilde{\Omega}_{2}(t)$ as

$$
\begin{aligned}
& \widetilde{\Omega}_{1}(t)=\zeta_{11} e^{-\left[\left(t-\tau_{11}\right) / \chi_{11}\right]^{2}}+\zeta_{12} e^{-\left[\left(t-\tau_{12}\right) / \chi_{12}\right]^{2}}, \\
& \widetilde{\Omega}_{2}(t)=\zeta_{21} e^{-\left[\left(t-\tau_{21}\right) / \chi_{21}\right]^{2}}+\zeta_{22} e^{-\left[\left(t-\tau_{22}\right) / \chi_{22}\right]^{2}},
\end{aligned}
$$

for $\Omega_{1}(t)$ and $\Omega_{2}(t)$, respectively, where,

$$
\begin{aligned}
& \zeta_{11}=-3.194 / T, \zeta_{12}=-1.275 / T, \zeta_{21}=3.194 / T, \zeta_{22}=1.275 / T, \\
& \tau_{11}=0.4396 T, \tau_{12}=0.2159 T, \tau_{21}=0.5604 T, \tau_{22}=0.7841 T, \\
& \chi_{11}=0.2476 T, \chi_{12}=0.1581 T, \chi_{21}=0.2476 T, \chi_{22}=0.1581 T .
\end{aligned}
$$

Here, $\zeta_{\alpha \beta}(\alpha, \beta=1,2)$ is the pulse amplitude of the $\beta$ th component in pulse $\Omega_{\alpha}(t), \tau_{\alpha \beta}$ describes the extreme point of the $\beta$ th component in pulse $\Omega_{\alpha}(t)$, and $\chi_{\alpha \beta}$ controls the width of the $\beta$ th component in pulse $\Omega_{\alpha}(t)$. To compare $\Omega_{1}(t)\left(\Omega_{2}(t)\right)$ and $\widetilde{\Omega}_{1}(t)\left(\widetilde{\Omega}_{2}(t)\right)$, we plot $\left|\Omega_{1}(t)\right|\left(\Omega_{2}(t)\right)$ and $\left|\widetilde{\Omega}_{1}(t)\right|\left(\widetilde{\Omega}_{2}(t)\right)$ versus $t / T$ in Fig. 1 (a) (Fig. 1 (b)). Seen from Fig. $1, \Omega_{1}(t)\left(\Omega_{2}(t)\right)$ and $\widetilde{\Omega}_{1}(t)\left(\widetilde{\Omega}_{2}(t)\right)$ are very close to each other. Besides, the pulse amplitude $\widetilde{\Omega}_{0}=\max _{0 \leq t \leq T}\left\{\widetilde{\Omega}_{1}(t), \widetilde{\Omega}_{2}(t)\right\}$ is only about 3.5/T. Moreover, the population $P_{j}(t)=\left|\left\langle j \mid \psi_{0}(t)\right\rangle\right|^{2}$ of state $|j\rangle(j=1,2,3)$ is plotted in Fig. 2. As shown in Fig. 2(a), $P_{3}$ increases from 0 
(a)

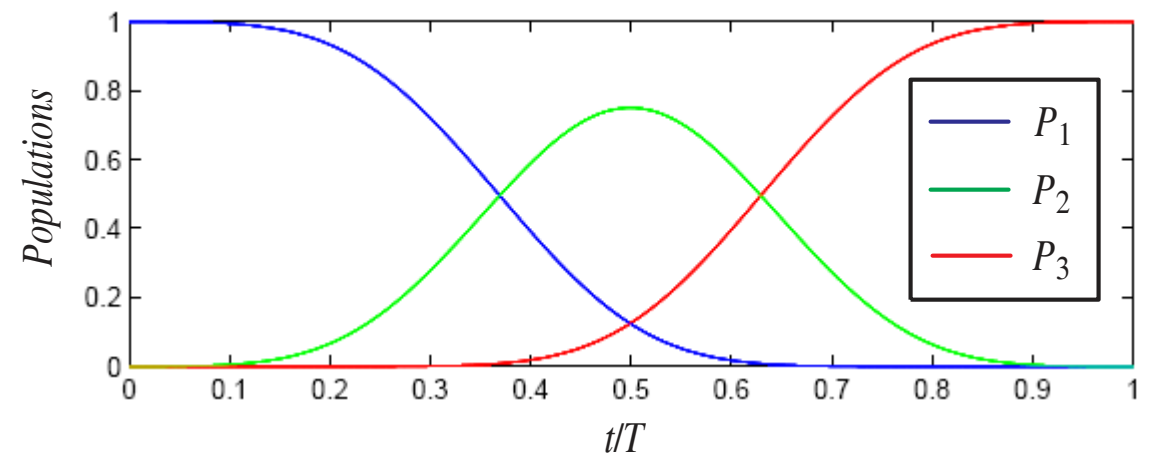

(b)

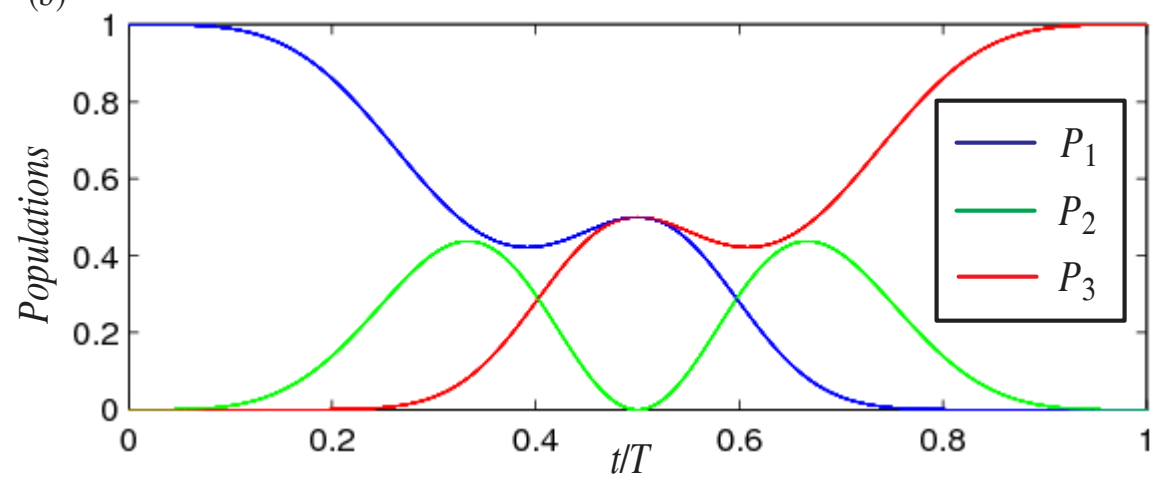

(c)

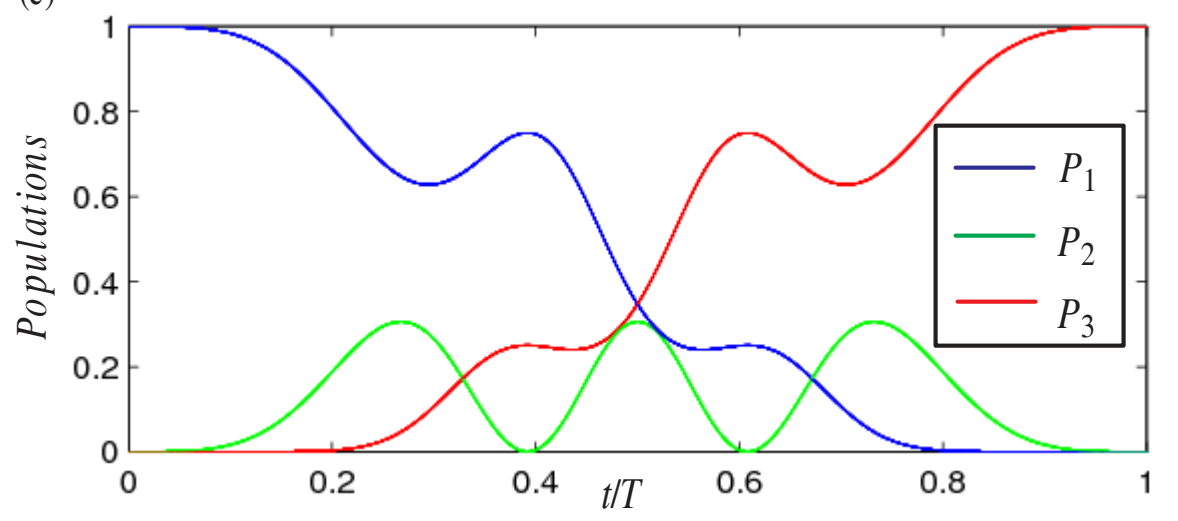

FIG. 2: The population $P_{j}$ state of $|j\rangle$ versus $t / T$ : (a) $\varphi(T)=\pi, \kappa=1 / 2$; (b) $\varphi(T)=2 \pi, \kappa=1 / 4$; (c) $\varphi(T)=3 \pi, \kappa=1 / 6$.

to 1 during the evolution, so the quantum transfer $|1\rangle \rightarrow|3\rangle$ can be achieved successfully. This proves that the method of the scheme and the replacing pulses in Eq. (15) are both effective. Fig. 2(a) also shows that $P_{2}$, the population of the intermediate state $|2\rangle$, reaches its maximal value $P_{2 \max }=2 \kappa-\kappa^{2}=0.75$ at $t=T / 2$. If we want to decrease $P_{2}$, we can increase $|\varphi(T)|$, so that a smaller $\kappa$ can be chosen. For example, if we choose $\varphi(T)=2 \pi$, then $\kappa=1 / 4$ can be adopted, so the maximal value of $P_{2}$ is $P_{2 \max }=2 \kappa-\kappa^{2}=0.4375$ (See 
Fig. 2(b)); if we choose $\varphi(T)=3 \pi$, then $\kappa=1 / 6$ is available, so the maximal value of $P_{2}$ is $P_{2 \max }=2 \kappa-\kappa^{2}=0.3056$ (See Fig. 2(c)). However, increasing $|\varphi(T)|$ requires us to increase the maximal value of $|\dot{\varphi}|$, since $\max _{0 \leq t \leq T}|\dot{\varphi}(t)| \geq|\varphi(T)| / T$ (See Table I). As a result, $\widetilde{\Omega}_{0}$ should also be increased. To have a relative high evolution speed, the product $\widetilde{\Omega}_{0} \times T$ (of the pulse amplitude $\widetilde{\Omega}_{0}$ and the total interaction time $T$ ) is the smaller the better. Because when $\widetilde{\Omega}_{0}$ is fixed (e.g. $\widetilde{\Omega}_{0}$ reaches the upper limit of the system), a smaller product $\widetilde{\Omega}_{0} \times T$ means a short interaction time $T$. On the other hand, in some cases, $P_{2}$ is required to be restrained in order to decrease the dissipation. Therefore, in real systems, one should choose a suitable value of control parameters for higher evolution speed and less dissipation.

Table I. The pulse amplitude $\widetilde{\Omega}_{0}$ and the maximal value of intermediate state's

\begin{tabular}{ccc} 
population $P_{2 \max }$ with corresponding $|\varphi(T)|$. \\
\hline \hline$|\varphi(T)|$ & $\widetilde{\Omega}_{0}$ & $P_{2 \max }$ \\
\hline$\pi$ & $3.5 / T$ & 0.75 \\
$2 \pi$ & $6.2 / T$ & 0.4375 \\
$3 \pi$ & $8.0 / T$ & 0.3056 \\
$4 \pi$ & $9.5 / T$ & 0.2344 \\
$5 \pi$ & $10.7 / T$ & 0.1900 \\
$6 \pi$ & $11.8 / T$ & 0.1597 \\
$7 \pi$ & $12.8 / T$ & 0.1378 \\
\hline \hline
\end{tabular}

Now, we would like to show that the quantum transfer can be significantly accelerated by using the present scheme. As a comparison, the stimulated Raman adiabatic passage (STIRAP) is also exploited to implement the quantum transfer. According to STIRAP method, the system evolves through the dark state $\left|\Psi_{\text {dark }}(t)\right\rangle=\frac{1}{\sqrt{\Omega_{1}^{2}(t)+\Omega_{2}^{2}(t)}}\left(\Omega_{2}(t)|1\rangle-\right.$ $\left.\Omega_{1}(t)|3\rangle\right)$ of Hamiltonian $H_{0}(t)$ shown in Eq. (1). By setting boundary condition

$$
\lim _{t \rightarrow-\infty}=\frac{\Omega_{1}(t)}{\Omega_{2}(t)}=0, \lim _{t \rightarrow+\infty}=\frac{\Omega_{2}(t)}{\Omega_{1}(t)}=0,
$$

one can design the Rabi frequencies $\Omega_{1}$ and $\Omega_{2}$ as following

$$
\begin{aligned}
& \Omega_{1}(t)=\Omega_{0} \exp \left[-\left(\frac{t-t_{0}-T / 2}{t_{c}}\right)^{2}\right], \\
& \Omega_{2}(t)=\Omega_{0} \exp \left[-\left(\frac{t+t_{0}-T / 2}{t_{c}}\right)^{2}\right],
\end{aligned}
$$




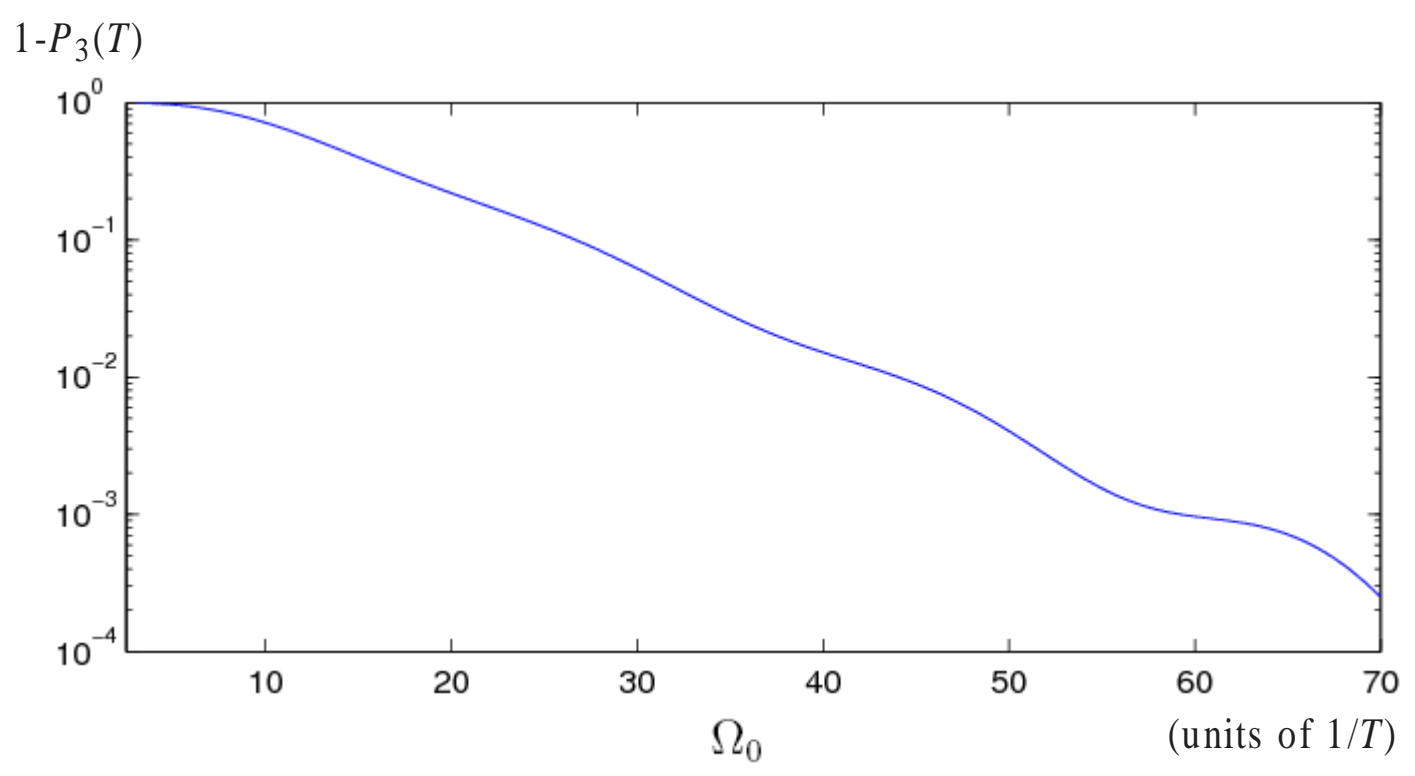

FIG. 3: $1-P_{3}(T)$ versus $\Omega_{0}$ for the STIRAP method.

where $\Omega_{0}$ denotes the pulse amplitude for STIRAP, $t_{0}$ and $t_{c}$ are two related parameters. Setting $t_{0}=0.15 T$ and $t_{c}=0.2 T$, Rabi frequencies $\Omega_{1}$ and $\Omega_{2}$ can well satisfy the boundary condition in Eq. (17). We plot $1-P_{3}(T)$ versus $\Omega_{0}$ for the STIRAP method in Fig. 3. As shown in Fig. 3, with STIRAP, for obtaining an enough high population of state $|3\rangle$, one should have $\Omega_{0} \geq 45 / T$. When $\Omega_{0}=70 / T, 1-P_{3}(T)$ is 0.0002 . For the present scheme, we have $1-P_{3}(T) \leq 3.714 \times 10^{-5}$ with $\widetilde{\Omega}_{0}=3.5 / T$. But for STIRAP, when $\Omega_{0}=3.5 / T$, $1-P_{3}(T)=0.9906$ due to the great violation of the adiabatic condition. As we mentioned above in this section, for a relatively high evolution speed, the product of the pulse amplitude and the total interaction time is the smaller the better. Therefore, using the present scheme, the quantum transfer can be significantly accelerated.

At the end of this section, let us check the robustness of the scheme with some numerical simulations. Firstly, we would like to show the robustness of the scheme against the parameters' errors caused by the imperfect operations. Here, we consider the errors $\delta T, \delta \widetilde{\Omega}_{1}$ and $\delta \widetilde{\Omega}_{2}$ of the total interaction time $T$, the Rabi frequencies of pulses $\widetilde{\Omega}_{1}$ and $\widetilde{\Omega}_{2}$, respectively. Before we perform the numerical simulations, we assume that $T^{\prime}=T+\delta T$ is the erroneous total interaction time. $P_{3}\left(T^{\prime}\right)$ versus $\delta \widetilde{\Omega}_{1} / \widetilde{\Omega}_{1}$ and $\delta \widetilde{\Omega}_{2} / \widetilde{\Omega}_{2}$ are shown by the blue crosses and the solid-red line in Fig. 4, respectively. And $P_{3}\left(T^{\prime}\right)$ versus $\delta T / T$ is plotted by the dashed-green line in Fig. 4. Seen from the dashed-green line in Fig. 4, we find that 


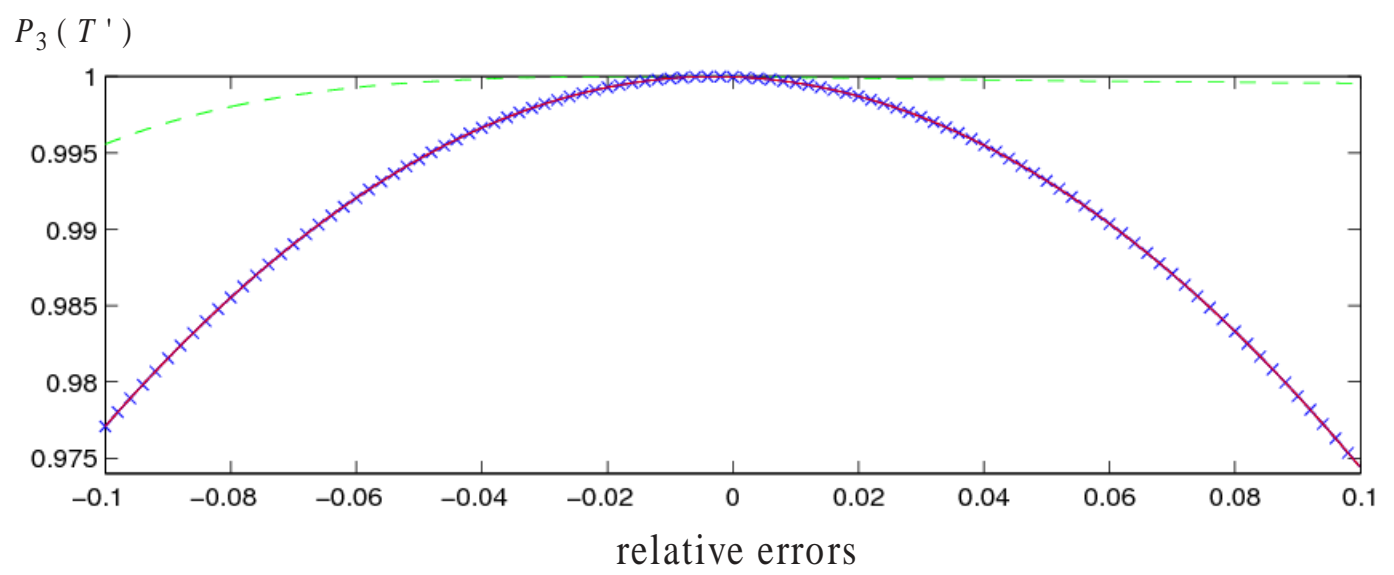

FIG. 4: $P_{3}\left(T^{\prime}\right)$ versus relative errors: $\delta \widetilde{\Omega}_{1} / \widetilde{\Omega}_{1}$ (blue crosses), $\delta \widetilde{\Omega}_{2} / \widetilde{\Omega}_{2}$ (solid-red line) and $\delta T / T$ (dashed-green line).

the scheme is quite robust against the timing errors, i.e., when $|\delta T / T| \leq 10 \%$, we have $P_{3}\left(T^{\prime}\right) \geq 0.9956$. Besides, according to the blue crosses and the solid-red line in Fig. 4, the influences of pulses' errors are larger than the timing error, however, $P_{3}(T)$ keeps higher than 0.9745 when $\left|\delta \widetilde{\Omega}_{1} / \widetilde{\Omega}_{1}\right| \leq 10 \%$ or $\left|\delta \widetilde{\Omega}_{2} / \widetilde{\Omega}_{2}\right| \leq 10 \%$. Therefore, the scheme holds nice robustness against the parameters' errors.

Secondly, let us analyze the robustness of the scheme against the decoherent factors. Here, we consider a superconducting (SC) qubit with $\Lambda$-type structure. For the SC qubit, there exists four decoherent factors: (i) the energy relaxation for the path $|2\rangle \rightarrow|1\rangle$ with energy relaxation rate $\Gamma_{1}$, (ii) the energy relaxation for the path $|2\rangle \rightarrow|3\rangle$ with energy relaxation rate $\Gamma_{2}$, (iii) the dephasing between energy levels $|2\rangle$ and $|1\rangle$ with dephasing rate $\Gamma_{\phi 1}$, (iv) the dephasing between energy levels $|2\rangle$ and $|3\rangle$ with dephasing rate $\Gamma_{\phi 2}$. Therefore, the evolution of the SC qubits can be described by a master equation in Lindblad form as following

$$
\dot{\rho}=i\left[\rho, H_{0}\right]+\sum_{l}\left[L_{l} \rho L_{l}^{\dagger}-\frac{1}{2}\left(L_{l}^{\dagger} L_{l} \rho+\rho L_{l}^{\dagger} L_{l}\right)\right],
$$

where, $L_{l}(l=1,2,3,4)$ is the Lindblad operator. Here, we have four Lindblad operators as

$$
\begin{aligned}
& L_{1}=\sqrt{\Gamma_{1}}|1\rangle\left\langle 2\left|, \quad L_{2}=\sqrt{\Gamma_{2}}\right| 3\right\rangle\langle 2|, \\
& L_{3}=\sqrt{\Gamma_{\phi 1}}(|2\rangle\langle 2|-| 1\rangle\langle 1|), \quad L_{4}=\sqrt{\Gamma_{\phi 2}}(|2\rangle\langle 2|-| 3\rangle\langle 3|) .
\end{aligned}
$$

We plot $P_{3}(T)$ versus $\Gamma_{1} / \widetilde{\Omega}_{0}$ and $\Gamma_{2} / \widetilde{\Omega}_{0}\left(\Gamma_{\phi 1} / \widetilde{\Omega}_{0}\right.$ and $\left.\Gamma_{\phi 2} / \widetilde{\Omega}_{0}\right)$ in Fig. $5(\mathrm{a})$ (Fig. $\left.5(\mathrm{~b})\right)$. Seen 

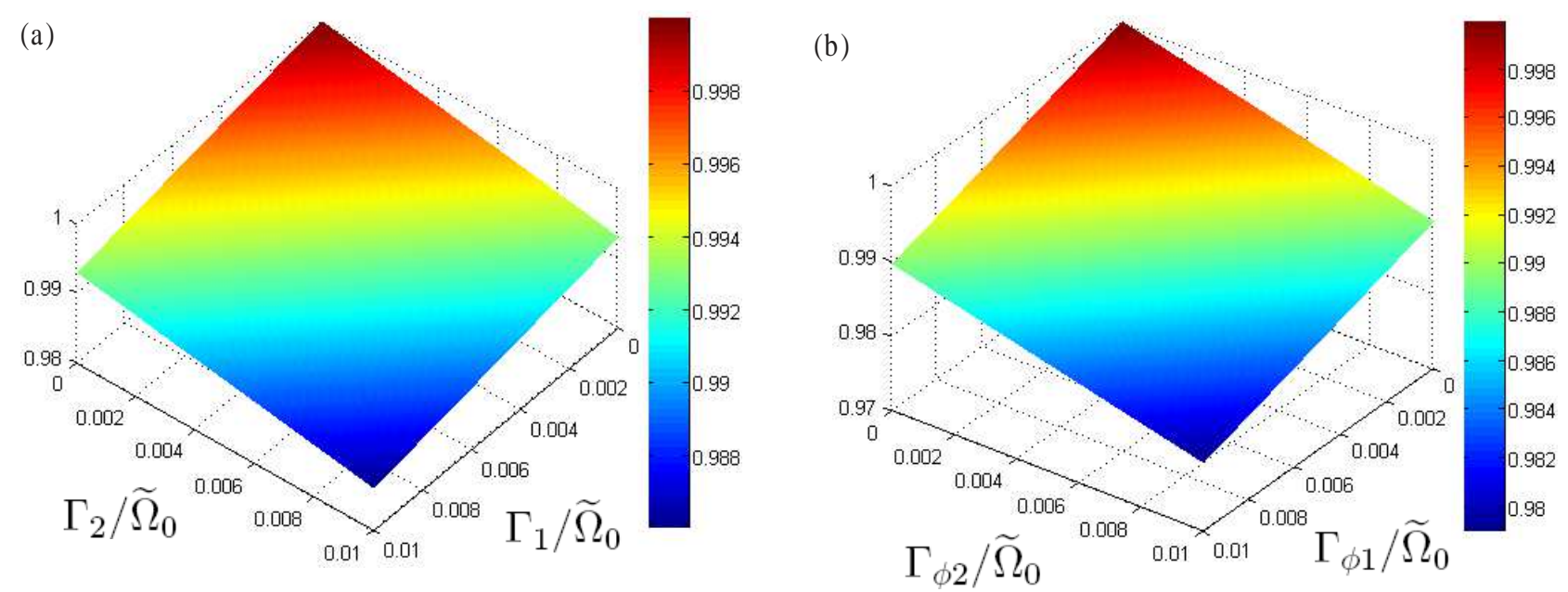

FIG. 5: (a) $P_{3}(T)$ versus $\Gamma_{1} / \widetilde{\Omega}_{0}$ and $\Gamma_{2} / \widetilde{\Omega}_{0}$. (b) $P_{3}(T)$ versus $\Gamma_{\phi 1} / \widetilde{\Omega}_{0}$ and $\Gamma_{\phi 2} / \widetilde{\Omega}_{0}$.

from Fig. 5 (a), $P_{3}(T)$ keeps higher than 0.986 for all $\Gamma_{1}$ and $\Gamma_{2}$ satisfying $\Gamma_{1} / \widetilde{\Omega}_{0} \leq 0.01$ and $\Gamma_{2} / \widetilde{\Omega}_{0} \leq 0.01$. According to Fig. $5(\mathrm{~b})$, we have $P_{3}(T) \geq 0.979$ when $\Gamma_{\phi 1} / \widetilde{\Omega}_{0} \leq 0.01$ and $\Gamma_{\phi 2} / \widetilde{\Omega}_{0} \leq 0.01$. Therefore, the scheme is also quite robust against energy relaxations and dephasings for SC qubits. However, the SC qubits is more sensitive to dephasings when using STIRAP, with pulses shown in Eq. (18), and parameters $\Omega_{0}=45 / T, t_{0}=0.15 T$, $t_{c}=0.20 T$, we have $P_{3}(T)=0.9561$ when $\Gamma_{\phi 1} / \Omega_{0}=\Gamma_{\phi 2} / \Omega_{0}=0.01$. According to Refs. [8, 16], for a multi-qubit system which has an effective Hamiltonian in $\Lambda$-type structure, the dephasings influence the SQ very much when using STIRAP. For example, Ref. [16] has shown that with STIRAP, when the ratio between dephasing and coupling strength is only 0.0001, the fidelity of the target state falls from 1 to about 0.85 . Therefore, the scheme may help to improve STIRAP for SC qubits.

\section{CONCLUSION}

In conclusion, we have proposed an alternative scheme to construct a shortcut to the adiabatic passage via picture transformation for quantum transfer in a system with three-level $\Lambda$-type structure. Different from previous works [60 73], the present scheme has its own feature. For example, schemes [66 68] have adopted a serials of iterative picture transformations, while here picture transformation is used only once. Besides, the ideas of schemes [66 68] are to cancel the transitions between the eigenstates of iterative Hamiltonian in each 
iterative picture, and the idea of scheme [73] suggests to cancel the transitions between a set of chosen dressed states. But for the present scheme, we directly study the dynamics of the three-level $\Lambda$-type system and the solution of the Schrödinger equation. Therefore, the present scheme consider the way to construct STAP from a different viewpoint. The present scheme has several advantages: (1) By choosing suitable control parameters, experimentally feasible pulses can be designed. (2) The quantum transfer can be achieved without any additional couplings. (3) Comparing with quantum transfer with adiabatic passages, the evolution is significantly sped up with present scheme.

Since the three-level $\Lambda$-type structure is very common in all kinds of physical systems including superconducting qubits [7, 8, 16], quantum dots or NV centers [11, 68], boson gas in longitudinal coordinate coupled waveguides [1 4, 60, 74, 75], atoms trapped in the cavities [5, 6, 9, 10, 12 15], etc., the present scheme can be a choice to construct STAP in these physical systems. Considering the potential applications of the present scheme in experiments, we hope the present scheme may be useful in quantum information field.

\section{ACKNOWLEDGEMENT}

This work was supported by the National Natural Science Foundation of China under Grants No. 11575045, No. 11374054 and No. 11675046, and the Major State Basic Research Development Program of China under Grant No. 2012CB921601.

[1] M. Ornigotti, G. D. Valle, T. T. Fernandez, A. Coppa, V. Foglietti, P. Laporta, S. Longhi, J. Phys. B 41 (2008) 085402.

[2] S. Longhi, Laser Photon. Rev. 3 (2009) 243-261.

[3] S. Longhi, J. Phys. B 44 (2010) 051001.

[4] A. A. Rangelov, N. V. Vitanov, Phys. Rev. A 85(2012) 055803.

[5] Y. H. Chen, Y. Xia, Q. Q. Chen, J. Song, Phys. Rev. A 89 (2014) 033856.

[6] M. Lu, Y. Xia, L. T. Shen, J. Song, N. B. An, Phys. Rev. A 89 (2014) 012326.

[7] J. Zhang, T. H. Kyaw, D. M. Tong, E. Sjövist, L. Kwek, Sci. Rep. 5 (2015) 18414.

[8] X. Wei, M. F. Chen, Quantum Inf. Process. 14 (2015) 2419-2433. 
[9] Y. H. Chen, Y. Xia, Q. Q. Chen, J. Song, Phys. Rev. A 91 (2015) 012325.

[10] X. B. Huang, Z. R. Zhong, Y. H. Chen, Quantum Inf. Process. 14 (2015) 4475-4492.

[11] X. K. Song, H. Zhang, Q. Ai, J. Qiu, F. G. Deng, New J. Phys. 18 (2016) 023001.

[12] Y. H. Chen, Y. Xia, J. Song, Q. Q. Chen, Sci. Rep. 5 (2016) 15616.

[13] Y. H. Chen, B. H. Huang, J. Song, Y. Xia, Opt. Comm. 380 (2016) 140-147.

[14] Z. Chen, Y. H. Chen, Y. Xia, J. Song, B. H. Huang, Sci. Rep. 6 (2016) 22202.

[15] W. J. Shan, Y. Xia, Y. H. Chen, J. Song, Quantum Inf. Process. 15 (2016) 2359-2376.

[16] J. L. Wu, C. Song, J. Xu, L. Yu, X. Ji, S. Zhang, Quantum Inf. Process. 15 (2016) 3663-3675.

[17] N. V. Golubev and A. I. Kuleff, Phys. Rev. A 90 (2014) 035401.

[18] S. B. Zheng, Phys. Rev. Lett. 90 (2003) 217901.

[19] S. B. Zheng, Phys. Rev. Lett. 95 (2005) 080502.

[20] P. Král, I. Thanopulos, M. Shapiro, Rev. Mod. Phys. 79 (2007) 53-77.

[21] K. Bergmann, H. Theuer, B. W. Shore, Rev. Mod. Phys. 70 (1998) 1003-1025.

[22] M. P. Fewell, B. W. Shore, K. Bergmann, Aust. J. Phys. 50 (1997) 281-308.

[23] N. V. Vitanov, T. Halfmann, B. W. Shore, K. Bergmann, Annu. Rev. Phys. Chem. 52 (2001) 763-809.

[24] M. Demirplak, S. A. Rice, J. Phys. Chem. A 107 (2003) 9937-9945.

[25] M. Demirplak, S. A. Rice, J. Chem. Phys. 129 (2008) 154111.

[26] M. V. Berry, J. Phys. A 42 (2009) 365303.

[27] E. Torrontegui, S. Ibáñez, S. Martínez-Garaot, M. Modugno, A. del Campo, D. Guéry-Odelin, A. Ruschhaupt, X. Chen, J. G. Muga, Adv. Atom. Mol. Opt. Phys. 62 (2013) 117-169.

[28] J. G. Muga, X. Chen, A. Ruschhaupt, D. Guéry-Odelin, J. Phys. B 42 (2009) 241001.

[29] X. Chen, A. Ruschhaupt, S. Schmidt, A. del Campo, D. Guéry-Odelin, J. G. Muga, Phys. Rev. Lett. 104 (2010) 063002.

[30] A. del Campo, M. G. Boshier, Sci. Rep. 2 (2012) 648.

[31] X. Chen, I. Lizuain, A. Ruschhaupt, D. Guéry-Odelin, J. G. Muga, Phys. Rev. Lett. 105 $123003(2010)$.

[32] A. del Campo, Phys. Rev. Lett. 111 (2013) 100502.

[33] X. Chen, E. Torrontegui, J. G. Muga, Phys. Rev. A 83 (2011) 062116.

[34] E. Torrontegui, S. Ibáñez, X. Chen, A. Ruschhaupt, D. Guéry-Odelin, J. G. Muga, Phys. Rev. A 83 (2011) 013415. 
[35] J. G. Muga, X. Chen, S. Ibáñez, I. Lizuain, A. Ruschhaupt, J. Phys. B 43 (2010) 085509.

[36] E. Torrontegui, X. Chen, M. Modugno, A. Ruschhaupt, D. Guéry-Odelin, J. G. Muga, Phys. Rev. A 85 (2012) 033605.

[37] S. Masuda, K. Nakamura, Phys. Rev. A 84 (2011) 043434.

[38] X. Chen, J. G. Muga, Phys. Rev. A 82 (2010) 053403.

[39] J. F. Schaff, P. Capuzzi, G. Labeyrie, P. Vignolo, New J. Phys. 13 (2011) 113017.

[40] X. Chen, E. Torrontegui, D. Stefanatos, J. S. Li, J. G. Muga, Phys. Rev. A 84 (2011) 043415.

[41] E. Torrontegui, X. Chen, M. Modugno, S. Schmidt, A. Ruschhaupt, J. G. Muga, New J. Phys. 14 (2012) 013031.

[42] A. del Campo, Eur. Phys. Lett. 96, 60005 (2011).

[43] A. Ruschhaupt, X. Chen, D. Alonso, J. G. Muga, New J. Phys. 14 (2012) 093040.

[44] J. F. Schaff, X. L. Song, P. Vignolo, G. Labeyrie, Phys. Rev. A 82 (2010) 033430.

[45] J. F. Schaff, X. L. Song, P. Capuzzi, P. Vignolo, G. Labeyrie, Eur. Phys. Lett. 93 (2011) 23001.

[46] X. Chen, J. G. Muga, Phys. Rev. A 86 (2012) 033405.

[47] A. C. Santos, M. S. Sarandy, Sci. Rep. 5 (2015) 15775.

[48] A. C. Santos, R. D. Silva, M. S. Sarandy, Phys. Rev. A 93 (2016) 012311.

[49] I. Hen, Phys. Rev. A 91 (2015) 022309.

[50] M. S. Sarandy, L. A. Wu, D. Lidar, Quantum Inf. Process. 3 (2004) 331-349.

[51] I. B. Coulamy, A. C. Santos, I. Hen, M. S. Sarandy, Front. ICT 3 (2016) 19.

[52] M. M. Rams, M. Mohseni, A. del Campo, New J. Phys. 18 (2016) 123034.

[53] S. Deffner, C. Jarzynski, A. del Campo, Phys. Rev. X 4 (2014) 021013.

[54] A. del Campo, Phys. Rev. A 84 (2011) 031606(R).

[55] Y. X. Du, Z. T. Liang, Y. C. Li, X. X. Yue, Q. X. Lv, W. Huang, X. Chen, H. Yan, S. L. Zhu, Nat. Commun. 7 (2016) 12479.

[56] S. An, D. Lv, A. del Campo, K. Kim, Nat. Commun. 7 (2016) 12999.

[57] L. Giannelli, E. Arimondo, Phys. Rev. A 89 (2014) 033419.

[58] S. Masuda, S. A. Rice, J. Phys. Chem. A 119 (2015) 3479.

[59] M. G. Bason, M. Viteau, N. Malossi, P. Huillery, E. Arimondo, D. Ciampini, R. Fazio, V. Giovannetti, R. Mannella, O. Morsch, Nat. Phys. 8 (2012) 147.

[60] S. Martínez-Garaot, E. Torrontegui, X. Chen, J. G. Muga, Phys. Rev. A 89 (2014) 053408. 
[61] T. Opatrný, K. Mømer, New J. Phys. 16 (2014) 015025.

[62] H. Saberi, T. Opatrný, K. Mømer, A. del Campo, Phys. Rev. A 90 (2014) 060301(R).

[63] E. Torrontegui, S. Martínez-Garaot, J. G. Muga, Phys. Rev. A 89 (2014) 043408.

[64] B. T. Torosov, G. D. Valle, S. Longhi, Phys. Rev. A 87 (2013) 052502.

[65] B. T. Torosov, G. D. Valle, S. Longhi, Phys. Rev. A 89 (2014) 063412.

[66] S. Ibáñez, X. Chen, E. Torrontegui, J. G. Muga, A. Ruschhaupt, Phys. Rev. Lett. 109 (2012) 100403.

[67] S. Ibáñez, X. Chen, J. G. Muga, Phys. Rev. A 87 (2013) 043402.

[68] X. K. Song, Q. Ai, J. Qiu, F. G. Deng, Phys. Rev. A 93 (2016) 052324.

[69] B. H. Huang, Y. H. Chen, Q. C. Wu, J. Song, Y. Xia, Laser Phys. Lett. 13 (2016) 105202.

[70] Y. H. Chen, Q. C. Wu, B. H. Huang, Y. Xia, J. Song, Phys. Rev. A 93 (2016) 052109.

[71] Y. H. Chen, J. Song, Y. Xia, Sci. Rep. 6 (2016) 38484.

[72] Y. H. Kang, Y. H. Chen, Q. C. Wu, B. H. Huang, Y. Xia, J. Song, Sci. Rep. 6 (2016) 30151.

[73] A. Baksic, H. Ribeiro, A. A. Clerk, Phys. Rev. Lett. 116 (2016) 230503.

[74] M. P. A. Fisher, P. B. Weichman, G. Grinstein, D. S. Fisher, Phys. Rev. B 40 (1989) 546-570.

[75] D. Jaksch, C. Bruder, J. I. Cirac, C. W. Gardiner, P. Zoller, Phys. Rev. Lett. 81 (1998) 3108-3111. 